\title{
Number of Unfavorable Intermediate-Risk Factors Predicts Pathologic Upstaging and Prostate Cancer Specific Mortality Following Radical Prostatectomy: Results from the SEARCH Database
}

Zachary S. Zumsteg, $\mathrm{MD}^{1,2, *}$ Zinan Chen, $\mathrm{BS}^{3}$ Lauren E. Howard, $\mathrm{MS}^{3}$ Christopher L. Amling, $\mathrm{MD}^{4}$ William J. Aronson, $\mathrm{MD}^{5,6}$ Matthew R. Cooperberg, MD, $\mathrm{MPH}^{7}$ Christopher J. Kane, $\mathrm{MD}^{8}$ Martha K. Terris, MD ${ }^{9,10}$ Daniel E. Spratt, MD ${ }^{11}$ Howard M. Sandler, MD ${ }^{1,2}$ Stephen J. Freedland, $\mathrm{MD}^{1,12}$

${ }^{1}$ Samuel Oschin Comprehensive Cancer Institute, Cedars-Sinai Medical Center, Los Angeles, California

${ }^{2}$ Department of Radiation Oncology, Cedars-Sinai Medical Center, Los Angeles, California ${ }^{3}$ Department of Biostatistics and Bioinformatics, Duke University School of Medicine, Durham, North Carolina

${ }^{4}$ Division of Urology, Oregon Health \& Sciences University, Portland, Oregon

5 Urology Section, Department of Surgery, Veterans Affairs Greater Los Angeles Healthcare System, Los Angeles, California

6 Department of Urology, UCLA School of Medicine, Los Angeles, California

7 Department of Urology, UCSF Helen Diller Family Comprehensive Cancer Center, San

Francisco, California

8 Urology Department, University of California San Diego Health System, San Diego, California 9 Section of Urology, Veterans Affairs Medical Center, Augusta, Georgia

10 Section of Urology, Medical College of Georgia, Augusta, Georgia 
11 Department of Radiation Oncology, University of Michigan, Ann Arbor, Michigan

12 Department of Urology, Cedars-Sinai Medical Center, Los Angeles, California

Correspondence: zachary.zumsteg@cshs.org, Department of Radiation Oncology, Cedars-Sinai Medical Center, 8700 Beverly Blvd., Los Angeles CA, 90048. Phone: 310-423-8077. Fax: 310423-6161.

Running Head: \# of Unfav Int-Risk Prostate CA Factors

Keywords: Prostate Cancer, Intermediate Risk, Unfavorable Intermediate Risk, Favorable Intermediate Risk

The authors have no conflicts of interest relevant to this manuscript to report. The SEARCH database is funded in part by the Department of Veterans Affairs and NIH K24 CA160653.

\section{Author Contributions:}

Conception/Design: ZZ, SJF

Statistical Analysis: ZC, LEH

Interpretation of Data: All authors

Manuscript writing and approval: All authors

\section{ABSTRACT}

Background: To validate and further improve the stratification of intermediate risk prostate cancer into favorable and unfavorable subgroups for patients undergoing radical prostatectomy.

Materials and Methods: The SEARCH database was queried for IR patients undergoing radical prostatectomy without adjuvant radiotherapy. UIR disease was defined any patient with at least 
one unfavorable risk factor (URF), including primary Gleason pattern 4, 50\% of more biopsy cores containing cancer, or multiple National Comprehensive Cancer Network IR factors.

Results: 1586 patients with IR prostate cancer comprised the study cohort. Median follow-up was 62 months. Patients classified as UIR were significantly more likely to have pathologic high risk features, such as Gleason score 8-10, pT3-4 disease, or lymph node metastases, than FIR patients $(\mathrm{p}<0.001)$. Furthermore, UIR patients had significantly higher rates of PSA-relapse $(\mathrm{PSA}$, hazard ratio $(\mathrm{HR})=1.89, \mathrm{P}<0.001)$ and distant metastasis $(\mathrm{DM}, \mathrm{HR}=2.92, \mathrm{P}=0.001)$, but no difference in prostate cancer-specific mortality (PCSM) or all-cause mortality in multivariable analysis. On secondary analysis, patients with $\geq 2$ URF had significantly worse PSA-RFS, DM, and PCSM than those with 0 or 1 URF. Moreover, $40 \%$ of patients with $\geq 2$ URF had high risk pathologic features.

Conclusions: Patients with UIR prostate cancer are at increased risk of PSA relapse, DM, and pathologic upstaging following prostatectomy. However, increased risk of PCSM was only detected in those with $\geq 2$ URF. This suggests that further refinement of the UIR subgroup may improve risk stratification.

\section{Introduction}

Prostate cancer is a clinically diverse entity comprising indolent tumors that can be safely observed, ${ }^{1,2}$ highly aggressive, treatment refractory tumors that produce lethal metastases, ${ }^{3,4}$ and a wide spectrum in between. In order to help distinguish between these possibilities and guide treatment recommendations, the National Comprehensive Cancer Network (NCCN) has developed a risk stratification system based on clinical tumor stage, biopsy Gleason, and pretreatment PSA. ${ }^{5}$ 
According to the NCCN system, patients with clinical tumor stage T2b-T2c, biopsy Gleason score 7, or a pretreatment PSA of 10 to $20 \mathrm{ng} / \mathrm{mL}$, but without other high risk features, are classified as intermediate-risk (IR). However, significant biologic and clinical heterogeneity exists within the IR group, ${ }^{6,7}$ and optimal management of this group remains controversial. ${ }^{8} \mathrm{~A}$ proposed modification to the NCCN system subdivides IR into favorable (FIR) and unfavorable (UIR) subgroups based on the primary Gleason pattern, percentage of positive prostate biopsy cores, and the number of NCCN intermediate-risk factors. ${ }^{6}$ The prognostic significance of these subgroups for patients undergoing radiation therapy has been validated in multiple independent datasets. $^{9-11}$

It has been suggested that the relatively worse outcomes for men with UIR prostate cancer are largely attributable to these patients having an increased risk of harboring occult high grade disease undetected by biopsy or more advanced tumor stage than appreciated clinically on digital rectal exam. ${ }^{12}$ However, the risk of pathologic upstaging for FIR versus UIR has not been reported to date, and the applicability of these classifications to patients undergoing prostatectomy is unknown. Additionally, a limitation of the current proposed definition of the UIR subgroup is that it is still a relatively broad classification encompassing approximately $60 \%$ of all IR patients. ${ }^{6,9}$ Thus, it is likely that significant heterogeneity exists even within those classified as UIR, and further stratification of this group is desirable. For these reasons, we analyzed a large cohort of men with IR prostate cancer undergoing radical prostatectomy across multiple medical centers in order to validate the FIR and UIR prostate cancer definitions in a surgical population, assess the risk of pathologic upstaging at time of surgery for each group, and more precisely define which UIR patients are at highest risk of distant metastasis (DM) and prostate cancer-specific mortality (PCSM) following radical prostatectomy. 


\section{Methods}

\section{Design and Participants}

After obtaining Institutional Review Board approval, patients undergoing radical prostatectomy from 1988 to 2013 at six Veterans Affairs Medical Centers (San Diego, West Los Angeles, and Palo Alto, CA; Durham and Asheville, NC, and Augusta, Georgia) were entered into the SEARCH database. ${ }^{13}$ Patients who received neoadjuvant therapy were excluded.

Intermediate-risk prostate cancer was defined according to NCCN guidelines as patients with clinical state T2b or T2c, biopsy Gleason of 7, or PSA 10-20ng/ml but without high-risk factors (PSA>20ng/ml, clinical stage T3a or higher, or biopsy Gleason>7). ${ }^{5}$ There were 2059 radical prostatectomy patients within the SEARCH database classified as NCCN intermediate-risk. Patients with missing outcome data $(n=16)$, missing biopsy information $(n=339)$, race $(n=13)$, pathological data $(n=41)$, or patients who received adjuvant radiation therapy $(n=63)$ were excluded, leaving 1586 patients for analysis.

NCCN IR patients were categorized into two mutually exclusive groups: UIR and FIR prostate cancer. UIR was defined as any IR patient with biopsy Gleason score 4+3, percentage of positive biopsy cores $(\mathrm{PPBC}) \geq 50 \%$, or multiple intermediate-risk factors (clinical state $\mathrm{T} 2 \mathrm{~b}$ or T2c, Biopsy Gleason of 7, or PSA 10-20ng/ml). All others were classified as FIR.

PSA-RFS after RP was defined as a single PSA greater than $0.2 \mathrm{ng} / \mathrm{ml}, 2$ values of $0.2 \mathrm{ng} / \mathrm{ml}$, or secondary treatment for an elevated postoperative PSA. ${ }^{14}$ Development of distant metastases (DM) was determined radiographically as evidence of prostate cancer outside of the prostate, seminal vesicles, or pelvic lymph nodes. Prostate cancer-specific death (PCSM) was defined as metastatic progressive CRPC at time of death with no obvious indication of another cause of death. All-cause mortality (ACM) included death from any cause. 


\section{Statistical Analysis}

Baseline characteristics of UIR and FIR patients were compared using t-tests or rank sum tests for continuous variables and chi-squared tests for categorical variables. We tested the differences between number of UIR factors ( 0 vs. 1 vs. 2-3) and pathological disease characteristics using chi-squared tests.

Cox proportional hazards were used to test the differences in time to PSA-RFS, DM, and ACM between UIR and FIR patients in both univariable and multivariable models (adjusted for clinical state $\leq$ T2a vs. T2b-T2c, pretreatment PSA $<10$ vs. 10-20ng/ml, age, race, and year of surgery). Comparisons of time to PCSM were analyzed using a competing-risk model with non-PCa death as the competing risk. We then repeated these analyses to test the associations between the number of UIR risk factors ( 0 vs. 1 vs. 2-3) and the outcomes listed above. Sensitivity analyses were conducted among patients with $\geq 8$ biopsy cores. Cumulative incidence curves were used to display the results graphically and differences between groups were tested using the log-rank test. Statistical significance was defined as $\mathrm{P}<0.05$. All analyses were performed using Stata v14.0.

\section{Results}

\section{Patient demographics}

In our study cohort $(n=1586), 961(60.6 \%)$ patients were classified as UIR and $625(39.4 \%)$ as FIR (Table 1). Median follow-up in the entire cohort was 62 months. In addition to characteristics used to define FIR and UIR (biopsy Gleason score, PPBC), UIR patients had significantly higher median pre-surgical PSA (7.6 vs. $6.2 \mathrm{ng} / \mathrm{mL}, \mathrm{p}<0.001)$ and higher clinical stage $(\mathrm{p}<0.001)$. UIR patients also had significantly increased risk of pathologic upstaging at surgery, with higher rates of extracapsular extension $(23.1 \%$ vs. $11.0 \%, \mathrm{p}<0.001)$, seminal 
vesicle invasion $(13.1 \%$ vs. $4.2 \%, \mathrm{p}<0.001)$, and pathologic Gleason score $8-10$ (13.3\% vs. $6.4 \%$, $\mathrm{P}<0.001)$. UIR patients were also somewhat more likely to have positive margins $(42.3 \% \mathrm{vs}$. $37.1 \%, \mathrm{p}=0.042$ ). Although pelvic lymph node dissection (PLND) was more likely to be performed for UIR patients, there was no significant difference in the rate of lymph node positivity in patients undergoing PLND ( $2.1 \%$ vs. $0.9 \%, \mathrm{P}=0.194$ by Fisher's exact test). In total, $31 \%$ of UIR patients had high risk pathologic features such as Gleason score 8-10, extracapsular extension, seminal vesicle invasion, or lymph node involvement present, compared to $15 \%$ of FIR patients. There was no significant difference between the UIR and FIR subgroups with respect to follow-up time, age, race, or year of surgery (all p-values >0.05).

\section{Clinical outcomes of UIR vs. FIR}

In univariable analysis (Table 2), UIR was associated with increased risk of PSA relapse $(\mathrm{HR}=1.89, \mathrm{P}<0.001)$ and $\mathrm{DM}(\mathrm{HR}=2.92, \mathrm{P}=0.001)$. There was a trend towards worse PCSM with UIR disease, but this did not reach statistical significance $(\mathrm{HR}=2.27, \mathrm{P}=0.057)$. After adjusting for other covariates in multivariable analysis (Table 2), PSA-RFS (HR=1.85, $\mathrm{P}<0.001)$ and DM $(\mathrm{HR}=2.95, \mathrm{P}=0.001)$ remained significantly worse for UIR patients, but PCSM was not significantly different $(\mathrm{HR}=1.94, \mathrm{P}=0.147)$. There was no association between UIR disease and ACM. The cumulative incidences of PSA relapse, DM, PCSM, and ACM in the FIR and UIR groups are shown in Figure 1.

\section{Risk of pathologic upstaging based on number of UIR factors}

Because in our cohort the UIR group comprised $60.6 \%$ of the total population, we chose to further stratify the UIR subgroup based on the total number of UIR factors present in each patient (Table 3). In our cohort, patients with 2-3 UIR factors were significantly more likely to have high risk features noted pathologically at surgery including Gleason score 8-10 (18.4\%), 
pathological stage T3-4 (40.2\%), extracapsular extension (31.1\%), and seminal vesicle invasion $(18.7 \%)$ versus patients with 0 or 1 UIR factors ( $\mathrm{p} \leq 0.001$ for all comparisons). Patient with $2-3$ UIR were also significantly more likely to have positive margins ( $\mathrm{p}=0.004)$. In total, $15 \%, 25 \%$, and $41 \%$ of patients with 0,1 , and 2-3 UIR factors, respectively, had high risk pathologic features including Gleason score 8-10, extracapsular extension, seminal vesicle invasion, or lymph node involvement. Similarly, $7 \%, 15 \%$, and $23 \%$ of these respective patients had very high risk pathologic features including primary Gleason pattern 5, seminal vesicle invasion, pT4 stage, or lymph node involvement.

\section{Clinical outcomes based on number of UIR factors}

With zero UIR factors as the reference, patients having either 1 or 2-3 UIF factors were at increased risk of PSA-RFS $\left(\mathrm{HR}_{\mathrm{UIR}=1}=1.57, \mathrm{P}<0.001 ; \mathrm{HR}_{\mathrm{UIR}=2-3}=2.48, \mathrm{P}<0.001\right)$ (table 4, Figure 2). This result remained significant and little changed in multivariable analysis $\left(\mathrm{HR}_{\mathrm{UIR}=1}=1.58, \mathrm{P}\right.$ $\left.<0.001 ; \mathrm{HR}_{\mathrm{UIR}=2-3}=2.42, \mathrm{P}<0.001\right)$. However, only patients with 2-3 UIF factors had significantly increased risk of DM and PCSM in both univariable $(\mathrm{HR}=4.50, \mathrm{P}<0.001 ; \mathrm{HR}=4.13$, $\mathrm{P}=0.001$, respectively) and multivariable models $(\mathrm{HR}=4.85, \mathrm{P}<0.001 ; \mathrm{HR}=3.99, \mathrm{P}=0.007$, respectively) compared to patients with zero UIR factors. By contrast, patients with only 1 UIR factor had similar risk of DM and PCSM to those with zero UIR factors ( $\mathrm{P}>0.05$ for all comparisons). ACM was similar amongst all groups.

\section{Results among patients with $\geq 8$ biopsy cores}

Given that the percentage of biopsy cores and primary Gleason pattern could be impacted by the total number of biopsy cores obtained, we performed a subgroup analysis only in those with $\geq 8$ biopsy cores $(\mathrm{N}=1340)$. Overall, results were similar to the entire cohort (Supplementary tables 1 and 2). UIR was associated with increased risk of PSA relapse and DM in both univariable and 
multivariable analyses, but not PCSM or ACM. Increasing numbers of UIR factors ( 0 vs. 1 vs. 23) were associated with increased risk of PSA-RFS and DM in both univariable and multivariable analyses. However, only patients with 2-3 UIR factors had increased risk of PCSM. In multivariable analysis, the hazard ratios for DM $(\mathrm{HR}=9.96, \mathrm{P}<0.001)$ and $\mathrm{PCSM}$ $(\mathrm{HR}=8.07, \mathrm{P}=0.015)$ were even stronger in the subgroup of patients with at least 8 biopsy cores when comparing patients with 2-3 UIR to those with 0 UIR factors, although confidence intervals were wide.

\section{Discussion}

In this study, we confirmed that, similar to patients undergoing radiotherapy, patients treated with radical prostatectomy with UIR prostate cancer have worse PSA-RFS and DM compared to those with FIR disease. However, there was no significant difference in PCSM in multivariable analyses when comparing these subgroups, contrary to what has been reported previously in definitive radiotherapy series. ${ }^{6,9,10}$ This is may be attributable to the relatively short follow-up (63 months) for patients in our dataset, especially given the long natural history of IR prostate cancer. However, an alternative explanation is that salvage radiotherapy, administered nearly twice as frequently to patients with UIR prostate cancer as those with FIR disease in our cohort ( $27 \%$ vs. $15 \%, \mathrm{p}<0.001)$, is efficacious in delaying or preventing subsequent PCSM for patients experiencing biochemical relapse following prostatectomy and helped mask differences in outcomes between these groups. ${ }^{15}$ In either case, we hypothesized that patients with multiple adverse risk factors may represent the proportion of UIR patients at highest risk of DM and PCSM. In fact, patients with 2 or more UIR factors had more than four times the risk of DM and of PCSM as patients with FIR prostate cancer, whereas patients with exactly 1 UIR factor had no significant difference in DM and PCSM as those with FIR disease. This suggests that significant 
heterogeneity exists even within the UIR subgroup, and that the worse prognosis harbored by this group is primarily driven by those with multiple unfavorable risk factors. Moreover, our results suggest a single unfavorable risk factor in isolation may not significantly affect prognosis for those with IR disease. However, given the relatively short follow-up time of our study, further follow-up is required to support these conclusions.

The risk of pathologic upstaging was substantial in our series for patients with UIR prostate cancer, with $31 \%$ of patients having high risk pathologic features such as Gleason score 8-10, extracapsular extension, seminal vesicle invasion, or lymph node involvement present. The risk of pathologic upstaging was even higher for patients with 2 or more UIR factors, with $41 \%$ having high risk features and 23\% have primary Gleason pattern 5, pT3b-T4 disease, both considered NCCN very high risk factors, ${ }^{3}$ or lymph node positivity. Thus, many patients with 2 or more UIR have occult high risk disease that may go undetected prior to surgery due to biopsy sampling error, the poor sensitivity of clinical staging via digital rectal exam, or both. Therefore, these patients may be candidates for clinical investigations studying additional presurgical evaluation to improve risk classification. For example, image-guided biopsy using a multiparametric MRI/TRUS fusion platform may represent a promising approach to ensure adequate sampling of prostate regions that are radiographically suspicious for high grade disease) or increase sampling of suspicious lesions areas often undersampled using standard TRUSguided biopsy, such as the anterior prostate. ${ }^{16-18}$ Additionally, preoperative MRI may increase detection of extracapsular extension and seminal vesicle invasion in comparison to digital rectal exam alone. However, although MRI has high specificity for extraprostatic extension, it has limited and variable sensitivity of approximately $60 \% .{ }^{19}$ Further, the clinical utility of such approaches in terms of guiding therapeutic decisions is investigational at this point, and it 
remains to be seen whether additional imaging modalities can improve risk stratification, and more importantly, improve the ability of a physician to make therapeutic recommendations beyond the standard clinical variables utilized in the our study.

It should be noted that increasing number of UIR factors was associated with increased risk of surgical margin involvement, with positive margins seen $37 \%, 39 \%$, and $48 \%$ of patients with 0 , 1 , and 2 or more UIR factors, respectively ( $\mathrm{p}=0.004)$. Therefore, we can't completely rule out the possibility that these differences in surgical margin status could have impacted our results, in addition to other clinicopathologic factors. However, we think that the differences in margin status are more likely to be a result of the higher risk of extraprostatic extension observed in patients with multiple UIR factors, rather than differences in surgical quality between these subgroups. Further, a recent large, multi-institutional study found that although positive surgical margins increased the risk of biochemical recurrence, there was no association with PCSM, ${ }^{20}$ making it unlikely that the increased risk of DM and PCSM noted in those with 2 or more UIR factors was related in any way to margin status. It is also notable that the surgical margin positivity rate in our study was somewhat higher than has been reported in some other series..$^{20,21}$ This could be attributable to differences in surgical technique, surgeon experience, pathologic margin evaluation procedures, baseline risk of extraprostatic extension in the respective cohorts, or other factors.

Our results validate in surgical patients the favorable versus unfavorable classifications of intermediate-risk prostate cancer that have been proposed previously for patients undergoing radiotherapy. ${ }^{6,8-11}$ However, this study also has several unique aspects and strengths that extend these prior findings. This study is the first to our knowledge to demonstrate increased risk of pathologic upstaging with UIR prostate cancer in comparison to FIR. Further, because this is a 
surgical population that did not receive neoadjuvant androgen deprivation, our study avoids one of the major confounding variables that limited conclusions in previous studies from radiotherapy cohorts with intermediate-risk disease. Additionally, our data demonstrate that there is heterogeneity even within the UIR group based on the number of UIR factors present, and that only patients with two or more UIR factor have worse PCSM than FIR patients. Another unique aspect of our study was the inclusion of a much higher proportion of African American patients (42\%) than previous studies of FIR and UIR prostate cancer. Although differences in biology, ${ }^{22}$ pathologic upstaging, ${ }^{23}$ and clinical outcomes have been described for prostate cancers developing in African Americans, ${ }^{24,25}$ our data provide confirmation that the FIR and UIR classifications are relevant in this population as well.

Several weaknesses of this study warrant further discussion. First, this is a retrospective study, with all attendant biases that accompany such investigations. Additionally, the median follow-up of our series is relatively short for outcomes such as DM and PCSM given the long natural history of intermediate-risk prostate cancer, and late occurring DM and PCSM events could affect the results with increased follow-up. Also, we also were not able to control for imbalances in the administration of salvage therapies such as radiation or androgen deprivation therapy given the complexity of such analyses,${ }^{26}$ and we can't rule out the possibility that differences in salvage therapy administration impacted the outcomes observed. Additionally, other factors not analyzed in our study, such as the percentage of Gleason pattern $4,{ }^{27}$ nuclear morphology, ${ }^{28}$ ductal histology, ${ }^{29}$ and tertiary Gleason pattern $5,{ }^{30}$ could also be important prognostic factors to consider for intermediate risk patients. Nomogram-based risk stratification, which was not analyzed in this study, may provide even more accurate risk prediction than categorical systems such as ours, given their continuous nature. Lastly, given that our study cohort was entirely 
treated within the VA Medical System, we can't be certain that these results are applicable to other populations treated in other medical settings, such as tertiary care academic centers or private community hospitals. Nevertheless, our study represents to our knowledge the largest investigation of clinical heterogeneity amongst intermediate-risk prostate cancer patients undergoing radical prostatectomy to date, and both supports and supplements results from prior studies in this population. ${ }^{6,9,10}$

In conclusion, our study validates that patients with UIR prostate cancer have increased risk of PSA relapse and DM in comparison to FIR patients following radical prostatectomy, which is likely at least in part due to their increased risk of harboring clinically undetected pathologic high grade disease or extraprostatic extension. Furthermore, patients with 2 or more UIR factors have increased risk and PCSM in comparison to patients with 0 or 1 UIR factor, even with shortterm follow-up. By contrast, patients with FIR and 1 UIR have similar rates of DM and PCSM, implying that the relatively poorer prognosis of UIR patients is driven by patients with multiple UIR factors. Recognition of the heterogeneity in clinical outcomes amongst the intermediate-risk population will hopefully aid in personalizing treatment recommendations for these patients.

\section{References}

1. Klotz L, Vesprini D, Sethukavalan P, et al: Long-term follow-up of a large active surveillance cohort of patients with prostate cancer. J Clin Oncol 33:272-7, 2015

2. Wilt TJ, Brawer MK, Jones KM, et al: Radical prostatectomy versus observation for localized prostate cancer. N Engl J Med 367:203-13, 2012

3. Sundi D, Wang VM, Pierorazio PM, et al: Very-high-risk localized prostate cancer: definition and outcomes. Prostate Cancer Prostatic Dis 17:57-63, 2014 
4. Beltran H, Tomlins S, Aparicio A, et al: Aggressive variants of castration-resistant prostate cancer. Clin Cancer Res 20:2846-50, 2014

5. Mohler JL, Kantoff PW, Armstrong AJ, et al: Prostate cancer, version 2.2014. J Natl Compr Canc Netw 12:686-718, 2014

6. Zumsteg ZS, Spratt DE, Pei I, et al: A new risk classification system for therapeutic decision making with intermediate-risk prostate cancer patients undergoing doseescalated external-beam radiation therapy. Eur Urol 64:895-902, 2013

7. Cancer Genome Atlas Research N: The Molecular Taxonomy of Primary Prostate Cancer. Cell 163:1011-25, 2015

8. Zumsteg ZS, Zelefsky MJ: Short-term androgen deprivation therapy for patients with intermediate-risk prostate cancer undergoing dose-escalated radiotherapy: the standard of care? Lancet Oncol 13:e259-69, 2012

9. Keane FK, Chen MH, Zhang D, et al: The likelihood of death from prostate cancer in men with favorable or unfavorable intermediate-risk disease. Cancer 120:1787-93, 2014

10. Keane FK, Chen MH, Zhang D, et al: Androgen deprivation therapy and the risk of death from prostate cancer among men with favorable or unfavorable intermediate-risk disease. Cancer 121:2713-9, 2015

11. Raldow AC, Zhang D, Chen MH, et al: Risk Group and Death From Prostate Cancer: Implications for Active Surveillance in Men With Favorable Intermediate-Risk Prostate Cancer. JAMA Oncol 1:334-40, 2015

12. D'Amico AV: Personalizing the management of men with intermediate-risk prostate cancer. Eur Urol 64:903-4, 2013 
13. Moreira DM, Howard LE, Sourbeer KN, et al: Predicting bone scan positivity in nonmetastatic castration-resistant prostate cancer. Prostate Cancer Prostatic Dis 18:333-7, 2015

14. Freedland SJ, Sutter ME, Dorey F, et al: Defining the ideal cutpoint for determining PSA recurrence after radical prostatectomy. Prostate-specific antigen. Urology $61: 365-9,2003$

15. Stephenson AJ, Scardino PT, Kattan MW, et al: Predicting the outcome of salvage radiation therapy for recurrent prostate cancer after radical prostatectomy. J Clin Oncol $25: 2035-41,2007$

16. Schoots IG, Roobol MJ, Nieboer D, et al: Magnetic resonance imaging-targeted biopsy may enhance the diagnostic accuracy of significant prostate cancer detection compared to standard transrectal ultrasound-guided biopsy: a systematic review and meta-analysis. Eur Urol $68: 438-50,2015$

17. Siddiqui MM, Rais-Bahrami S, Truong H, et al: Magnetic resonance imaging/ultrasound-fusion biopsy significantly upgrades prostate cancer versus systematic 12core transrectal ultrasound biopsy. Eur Urol 64:713-9, 2013

18. Volkin D, Turkbey B, Hoang AN, et al: Multiparametric magnetic resonance imaging (MRI) and subsequent MRI/ultrasonography fusion-guided biopsy increase the detection of anteriorly located prostate cancers. BJU Int 114:E43-9, 2014

19. de Rooij M, Hamoen EH, Witjes JA, et al: Accuracy of Magnetic Resonance Imaging for Local Staging of Prostate Cancer: A Diagnostic Meta-analysis. Eur Urol, 2015

20. Stephenson AJ, Eggener SE, Hernandez AV, et al: Do margins matter? The influence of positive surgical margins on prostate cancer-specific mortality. Eur Urol 65:675-80, 2014 
21. Yossepowitch O, Briganti A, Eastham JA, et al: Positive surgical margins after radical prostatectomy: a systematic review and contemporary update. Eur Urol 65:303-13, 2014

22. Yamoah K, Johnson MH, Choeurng V, et al: Novel Biomarker Signature That May Predict Aggressive Disease in African American Men With Prostate Cancer. J Clin Oncol $33: 2789-96,2015$

23. Sundi D, Ross AE, Humphreys EB, et al: African American men with very lowrisk prostate cancer exhibit adverse oncologic outcomes after radical prostatectomy: should active surveillance still be an option for them? J Clin Oncol 31:2991-7, 2013

24. Faisal FA, Sundi D, Cooper JL, et al: Racial disparities in oncologic outcomes after radical prostatectomy: long-term follow-up. Urology 84:1434-41, 2014

25. Taksler GB, Keating NL, Cutler DM: Explaining racial differences in prostate cancer mortality. Cancer 118:4280-9, 2012

26. Kennedy EH, Taylor JM, Schaubel DE, et al: The effect of salvage therapy on survival in a longitudinal study with treatment by indication. Stat Med 29:2569-80, 2010

27. Cole AI, Morgan TM, Spratt DE, et al: Prognostic Value of Percent Gleason Grade 4 at Prostate Biopsy in Predicting Prostatectomy Pathology and Recurrence. J Urol 196:405-11, 2016

28. Veltri RW, Isharwal S, Miller MC, et al: Nuclear roundness variance predicts prostate cancer progression, metastasis, and death: A prospective evaluation with up to 25 years of follow-up after radical prostatectomy. Prostate 70:1333-9, 2010

29. Seipel AH, Delahunt B, Samaratunga H, et al: Ductal adenocarcinoma of the prostate: histogenesis, biology and clinicopathological features. Pathology 48:398-405, 2016

30. Patel AA, Chen MH, Renshaw AA, et al: PSA failure following definitive treatment of prostate cancer having biopsy Gleason score 7 with tertiary grade 5. JAMA 298:1533-8, 2007 
Figure Legends:

Figure 1. Cumulative incidence of PSA relapse (A), distant metastasis (B), prostate cancer specific mortality (C), and all-cause mortality (D) in favorable intermediate risk (FIR) and unfavorable intermediate risk prostate cancer patients from time of radical prostatectomy.

Figure 2. Cumulative incidence of PSA relapse (A), distant metastasis (B), prostate cancer specific mortality (C), and all-cause mortality (D) in patients with 0,1 , or $2-3$ unfavorable intermediate risk (UIR) factors. UIR factors are defined as Gleason score $4+3, \geq 50 \%$ positive biopsy cores, or more than one NCCN intermediate risk factor.

Table 1. Baseline clinical and pathological characteristics $(\mathrm{N}=1586)$

\begin{tabular}{|c|c|c|c|}
\hline & Favorable & Unfavorable & $\mathrm{P}$ value \\
\hline No. of Patient $(\%)$ & $625(39.4)$ & $961(60.6)$ & - \\
\hline PSA Follow-up mo, Median (IQR)* & $50.6(26.2,96.5)$ & $41.0(21.3,82.0)$ & $0.002^{1}$ \\
\hline Total Follow-up mo, Median (IQR)** & $63.5(34.2,115.2)$ & $61.4(31.1,110.1)$ & $0.247^{1}$ \\
\hline Age, yr, Mean (SD) & $62.1(6.1)$ & $61.9(6.2)$ & $0.459^{2}$ \\
\hline Race & & & $0.679^{3}$ \\
\hline White & $344(55.0)$ & $527(54.9)$ & \\
\hline Black & $265(42.4)$ & $402(41.8)$ & \\
\hline Other & $16(2.6)$ & $32(3.3)$ & \\
\hline Year of Surgery, Median (IQR) & $2008(2003,2012)$ & $2008(2003,2012)$ & $0.322^{1}$ \\
\hline Clinical T Stage, No. (\%) & & & $<0.001^{4}$ \\
\hline T1a-c & $409(65.4)$ & $505(52.6)$ & \\
\hline $\mathrm{T} 2$ & $20(3.2)$ & $26(2.7)$ & \\
\hline $\mathrm{T} 2 \mathrm{a}$ & $156(25.0)$ & $242(25.2)$ & \\
\hline $\mathrm{T} 2 \mathrm{~b}$ & $40(6.4)$ & $104(10.8)$ & \\
\hline $\mathrm{T} 2 \mathrm{c}$ & 0 & $84(8.7)$ & \\
\hline Biopsy Gleason Score (\%) & & & $<0.001^{4}$ \\
\hline$\leq 6$ & $201(32.2)$ & $107(11.1)$ & \\
\hline $3+4$ & $424(67.8)$ & $459(47.8)$ & \\
\hline $4+3$ & 0 & 395 (41.1) & \\
\hline PSA, Median (IQR) & $6.2(4.9,10.0)$ & $7.6(5.2,11.3)$ & $<0.001^{1}$ \\
\hline $\begin{array}{l}\text { Percentage Positive Biopsy Cores } \\
\text { Median (IQR) }\end{array}$ & & & $<0.001^{4}$ \\
\hline$<50 \%$ & $625(100)$ & 369 (38.4) & \\
\hline
\end{tabular}


Pathological Gleason Score (\%)

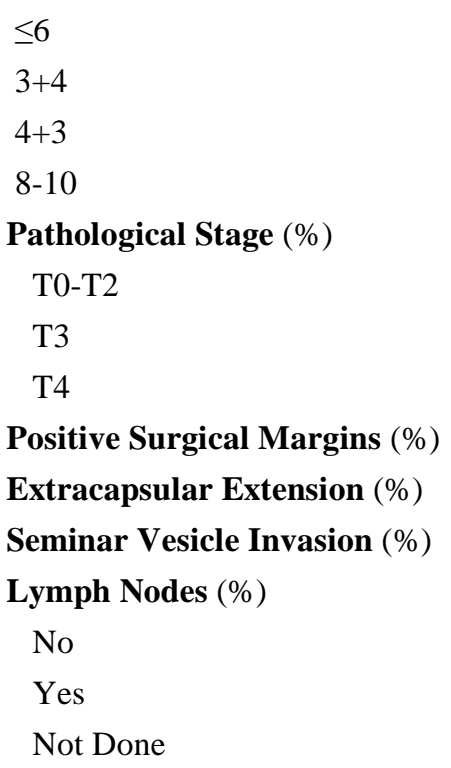

Number of Lymph Nodes Removed Median (IQR)

Received Salvage ADT (\%)

Received Salvage XRT (\%)

$\begin{array}{cc}181(29.0) & 125(13.0) \\ 306(49.0) & 457(47.6) \\ 98(15.6) & 251(26.1) \\ 40(6.4) & 128(13.3)\end{array}$

$534(85.4)$

$76(12.2)$

$15(2.4)$

$232(37.1)$

69 (11.0)

$26(4.2)$

$410(65.6)$

$5(0.8)$

$210(33.6)$

$4(2,8)$

$54(8.6)$

$96(15.4)$
$<0.001^{3}$

$<0.001^{3}$

$663(69.0)$

$264(27.5)$

$34(3.5)$

$406(42.3) \quad 0.042^{3}$

$222(23.1) \quad<0.001^{3}$

$126(13.1)<0.001^{3}$

$<0.001^{4}$

749 (77.9)

19 (2.0)

$193(20.1)$

$5(2,10)$

$0.009^{1}$

$189(19.7)<0.001^{3}$

$257(26.7) \quad<0.001^{3}$

Abbreviations: SD, standard deviation; ADT, androgen-deprivation therapy; PSA, prostate-specific antigen; XRT, radiation therapy

$\mathrm{P}$-value calculated using ${ }^{1}$ rank sum test ${ }^{2}$ student $\mathrm{t}$ test ${ }^{3}$ chi-squared test ${ }^{4}$ Fisher's exact test

*Reported among those who did not recur

**Reported among those who did not die

Table 2. Association between favorable vs. unfavorable intermediate-risk and prostate cancer outcomes

\begin{tabular}{lcccc}
\hline \multicolumn{2}{c}{ Univariable } & \multicolumn{2}{c}{ Multivariable* } \\
\hline \multicolumn{1}{c}{ HR $(95 \% \mathrm{CI})$} & $\mathrm{p}$-value & HR $(95 \% \mathrm{CI})$ & p-value \\
\hline PSA-RFS & & & & \\
\hline UIR vs FIR & $1.89(1.58,2.26)$ & $<0.001$ & $1.85(1.54,2.23)$ & $<0.001$ \\
$\begin{array}{l}\text { DM } \\
\text { UIR vs FIR }\end{array}$ & $2.92(1.55,5.47)$ & 0.001 & $2.95(1.55,5.63)$ & 0.001 \\
$\begin{array}{l}\text { PCSM } \\
\text { UIR vs FIR }\end{array}$ & $2.27(0.97,5.27)$ & 0.057 & $1.94(0.79,4.76)$ & 0.147 \\
$\begin{array}{l}\text { ACM } \\
\text { UIR vs FIR }\end{array}$ & $1.08(0.86,1.36)$ & 0.522 & $1.07(0.84,1.37)$ & 0.581
\end{tabular}

*Adjusted for: clinical tumor stage ( $\leq$ T2a vs. T2b-T2c), pretreatment PSA (<10 vs. 10-20), age, race, year of surgery and center.

Abbreviation: PSA-RFS: Prostate-specific antigen recurrence-free survival; DM: distant metastasis; PCSM: prostate cancer specific mortality; ACM: all-cause mortality

** Note: Out of 1586 patients, there were 567 recurrences, 63 distant metastases, 30 death of prostate cancer and 299 all-cause deaths

Table 3. Risk of pathologic upstaging stratified by number unfavorable intermediate-risk factors 


\begin{tabular}{lcccc}
\hline & Favorable & Unfavorable & P value \\
\hline & 0 UIR factors & 1 UIR factor & 2 or 3 UIR factors & \\
\hline No. of Patient (\%) & $625(39.4)$ & $598(37.7)$ & $363(22.9)$ & \\
Pathological Gleason Score (\%) & & & & $<0.001^{1}$ \\
$\quad \leq 6$ & $181(29.0)$ & $96(16.0)$ & $29(8.0)$ & \\
$3+4$ & $306(48.9)$ & $309(51.7)$ & $148(40.8)$ & \\
$4+3$ & $98(15.7)$ & $132(22.1)$ & $119(32.8)$ & \\
$8-10$ & $40(6.4)$ & $61(10.2)$ & $67(18.4)$ & \\
Pathological Stage $(\%)$ & & & & \\
T2 & $534(85.4)$ & $446(74.6)$ & $217(59.8)$ & \\
T3 & $76(12.2)$ & $129(21.6)$ & $135(37.2)$ & \\
T4 & $15(2.4)$ & $23(3.8)$ & $11(3.0)$ & \\
Positive Surgical Margins $(\%)$ & $232(37.1)$ & $233(39.0)$ & $173(47.7)$ & $0.004^{1}$ \\
Extracapsular Extension $(\%)$ & $69(11.0)$ & $109(18.2)$ & $113(31.1)$ & $<0.001^{1}$ \\
Seminar Vesicle Invasion $(\%)$ & $26(4.2)$ & $58(9.7)$ & $68(18.7)$ & $<0.001^{1}$ \\
Lymph Nodes $(\%)$ & & & & $<0.001^{2}$ \\
$\quad$ No & $410(65.6)$ & $436(72.9)$ & $313(86.2)$ & \\
$\quad$ Yes & $5(0.8)$ & $7(1.2)$ & $12(3.3)$ & \\
$\quad$ Not Done & $210(33.6)$ & $155(25.9)$ & $38(10.5)$ & \\
\hline
\end{tabular}

${ }^{1} \mathrm{P}$-value calculated using chi-squared test ${ }^{2}$ Fisher's exact test

Table 4. Association between number of UIR factors and prostate cancer outcomes

\begin{tabular}{|c|c|c|c|c|}
\hline & \multicolumn{2}{|c|}{ Univariable } & \multicolumn{2}{|c|}{ Multivariable* } \\
\hline & HR $(95 \%$ CI $)$ & p-value & HR $(95 \% \mathrm{CI})$ & p-value \\
\hline \multicolumn{5}{|l|}{ PSA-RFS } \\
\hline 0 UIR risk factors & Ref. & & Ref. & \\
\hline 1 UIR risk factor & $1.57(1.28,1.92)$ & $<0.001$ & $1.58(1.29,1.94)$ & $<0.001$ \\
\hline 2-3 UIR risk factors & $2.48(2.02,3.06)$ & $<0.001$ & $2.42(1.94,3.03)$ & $<0.001$ \\
\hline \multicolumn{5}{|l|}{ DM } \\
\hline 0 UIR risk factors & Ref. & & Ref. & \\
\hline 1 UIR risk factor & $1.89(0.92,3.86)$ & 0.082 & $1.98(0.96,4.09)$ & 0.065 \\
\hline 2-3 UIR risk factors & $4.50(2.31,8.77)$ & $<0.001$ & $4.85(2.39,9.85)$ & $<0.001$ \\
\hline \multicolumn{5}{|l|}{ PCSM } \\
\hline 0 UIR risk factors & Ref. & & Ref. & \\
\hline 1 UIR risk factor & $1.00(0.33,2.96)$ & 0.993 & $0.91(0.30,2.75)$ & 0.868 \\
\hline 2-3 UIR risk factors & $4.13(1.72,9.90)$ & 0.001 & $3.99(1.46,10.88)$ & 0.007 \\
\hline \multicolumn{5}{|l|}{$\mathbf{A C M}$} \\
\hline 0 UIR risk factors & Ref. & & Ref. & \\
\hline 1 UIR risk factor & $1.01(0.78,1.32)$ & 0.934 & $1.05(0.80,1.38)$ & 0.719 \\
\hline 2-3 UIR risk factors & $1.18(0.89,1.57)$ & 0.256 & $1.11(0.81,1.51)$ & 0.527 \\
\hline
\end{tabular}

*Adjusted for: clinical tumor stage ( $\leq$ T2a vs. T2b-T2c), pretreatment PSA ( $<10$ vs. 10-20), age, race, year of surgery and center.

Abbreviation: PSA-RFS: Prostate-specific antigen recurrence-free survival; DM: distant metastasis; PCSM: prostate cancer specific mortality; ACM: all-cause mortality

** Note: Out of 1586 patients, there were 567 recurrences, 63 distant metastases, 30 death of prostate cancer and 299 all-cause deaths 


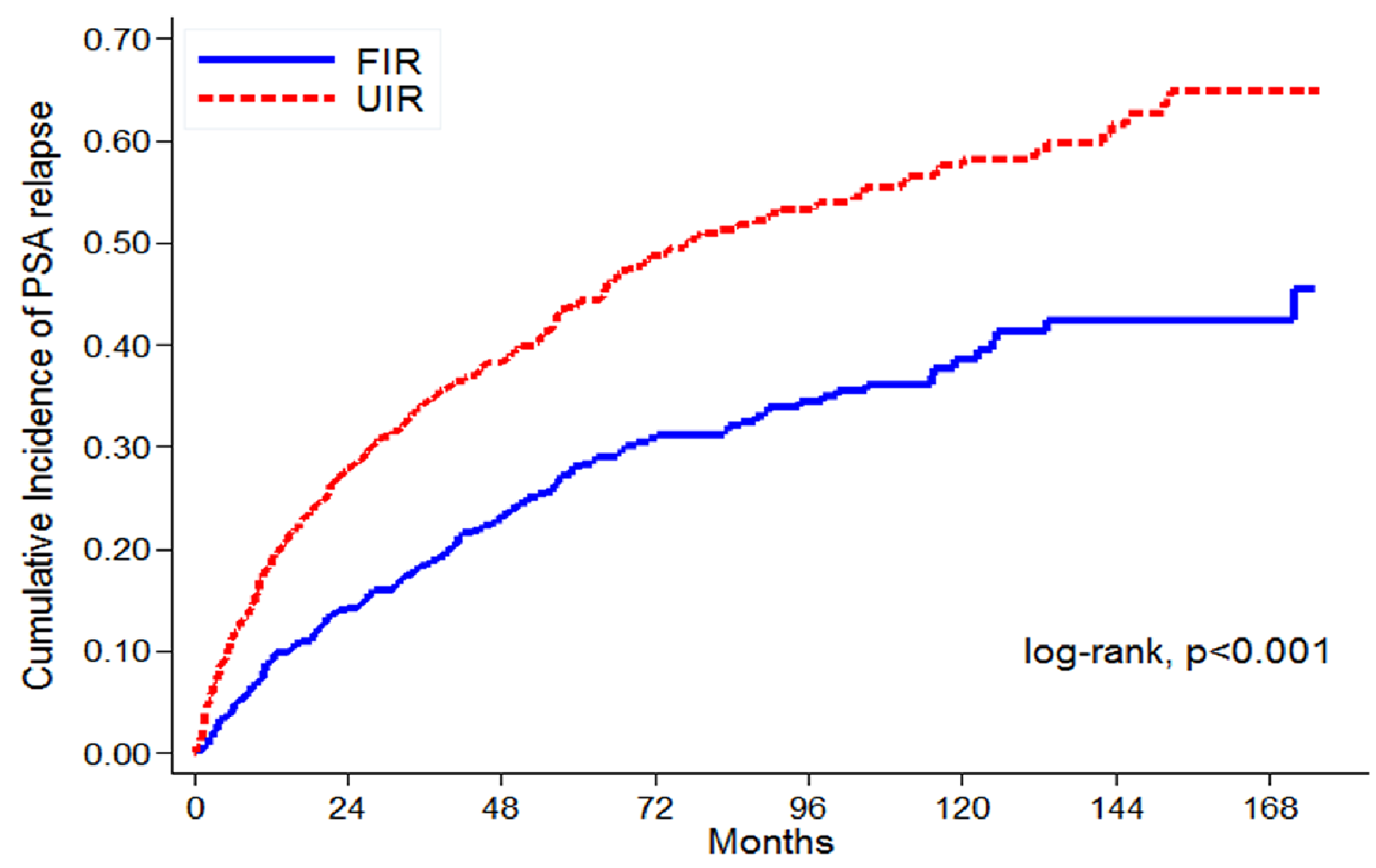

Fig 1A_300dpi . 


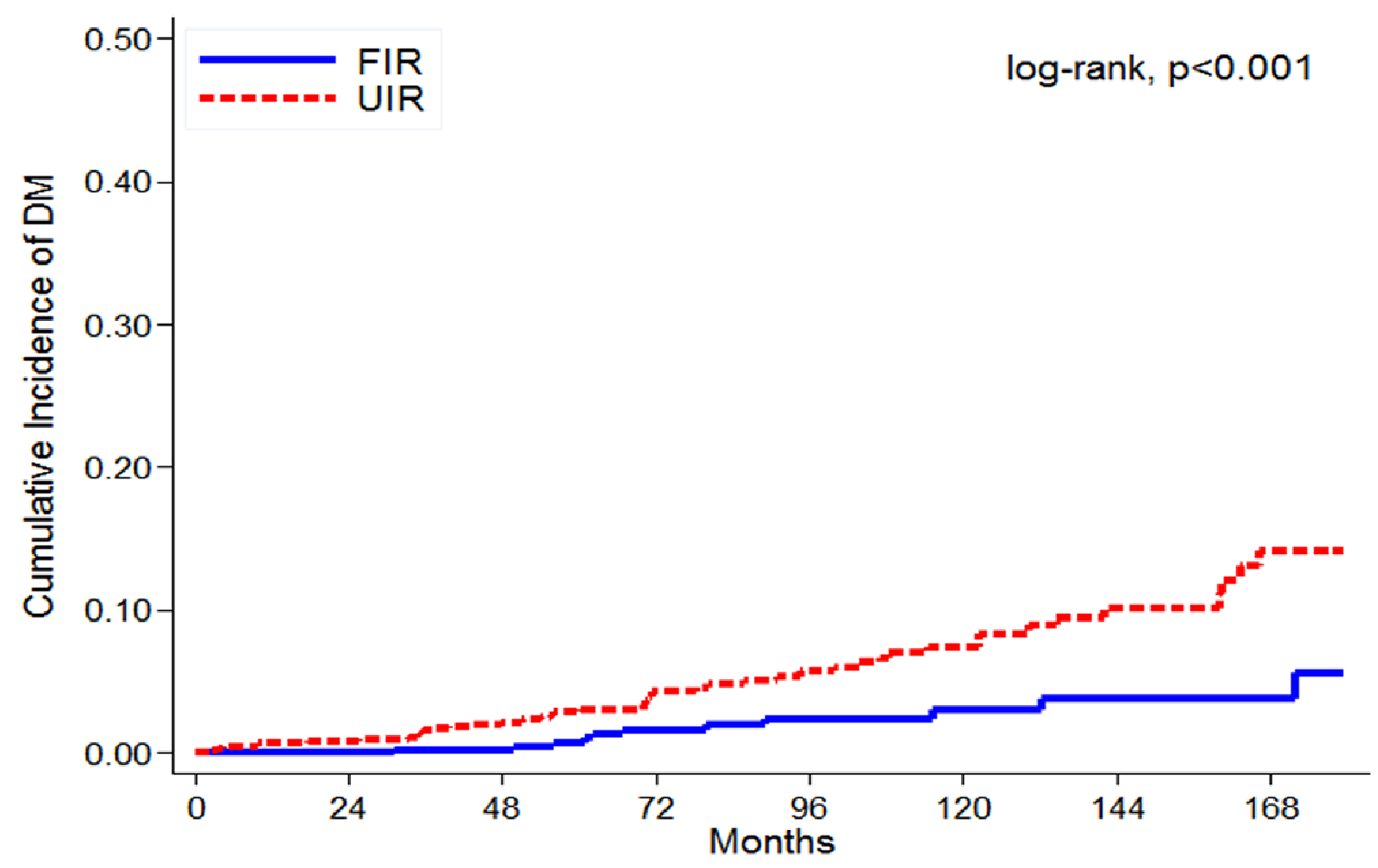

Fig 1B_300dpi . 


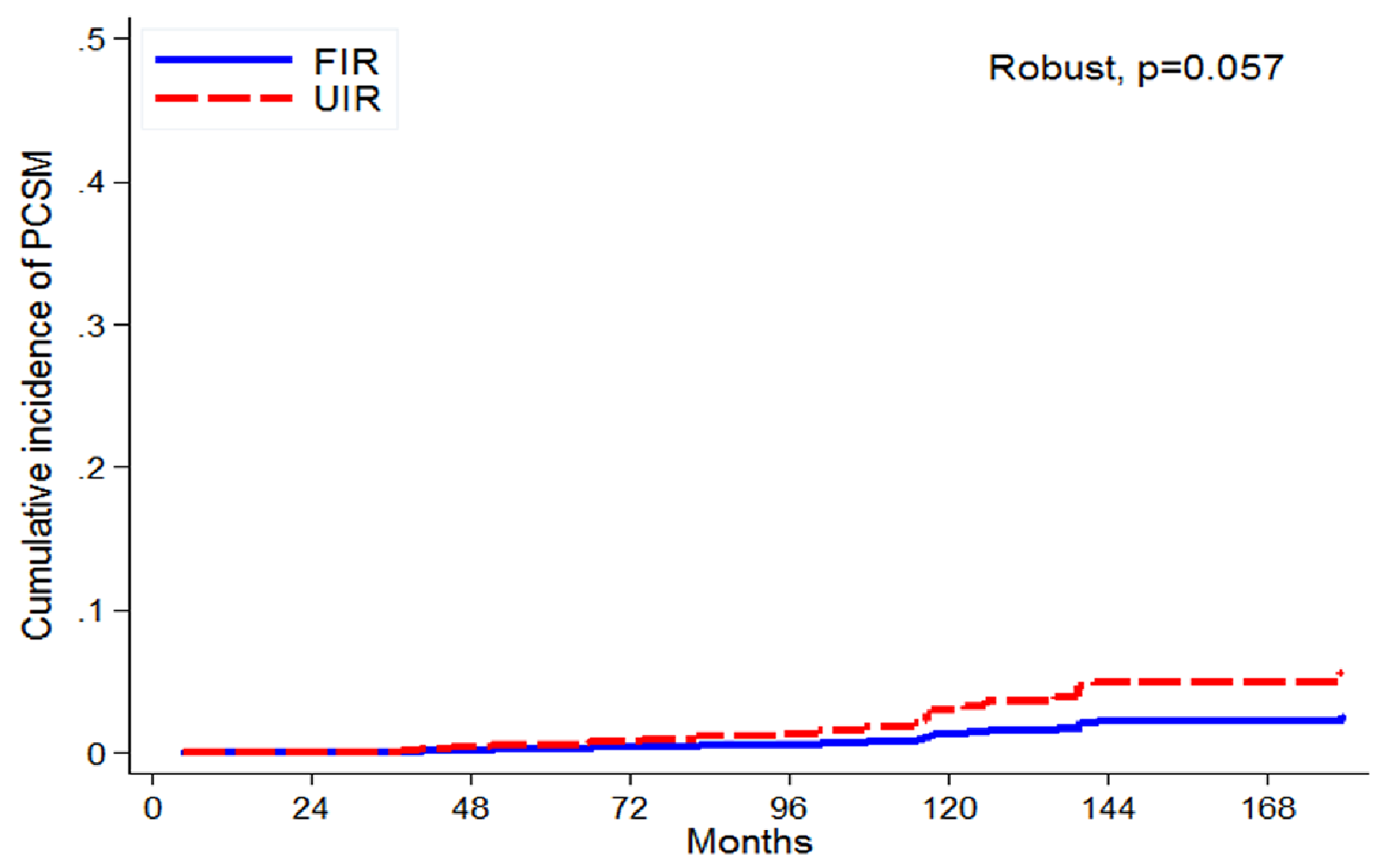

Fig 1C_300dpi . 


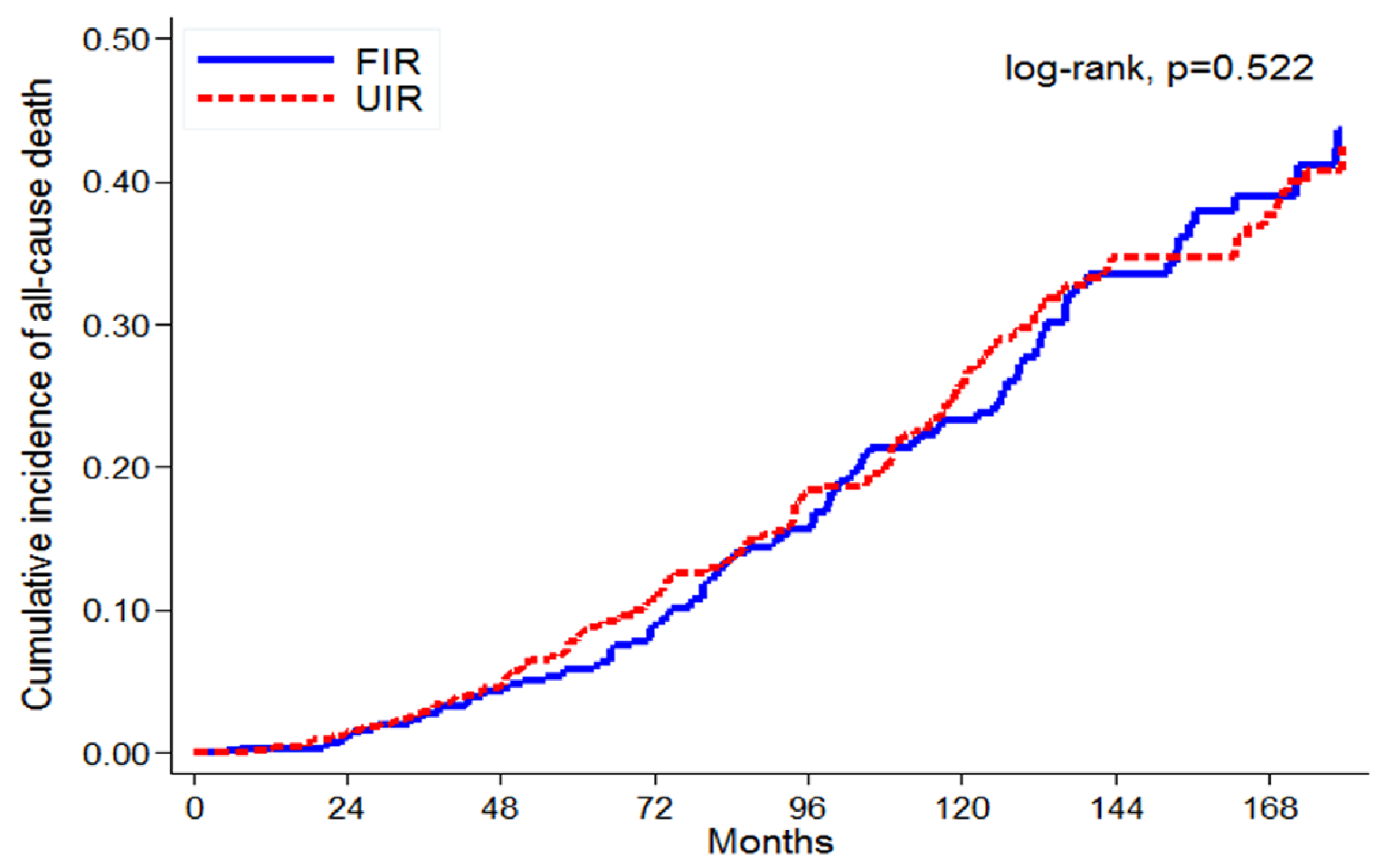

Fig 1D_300dpi . 


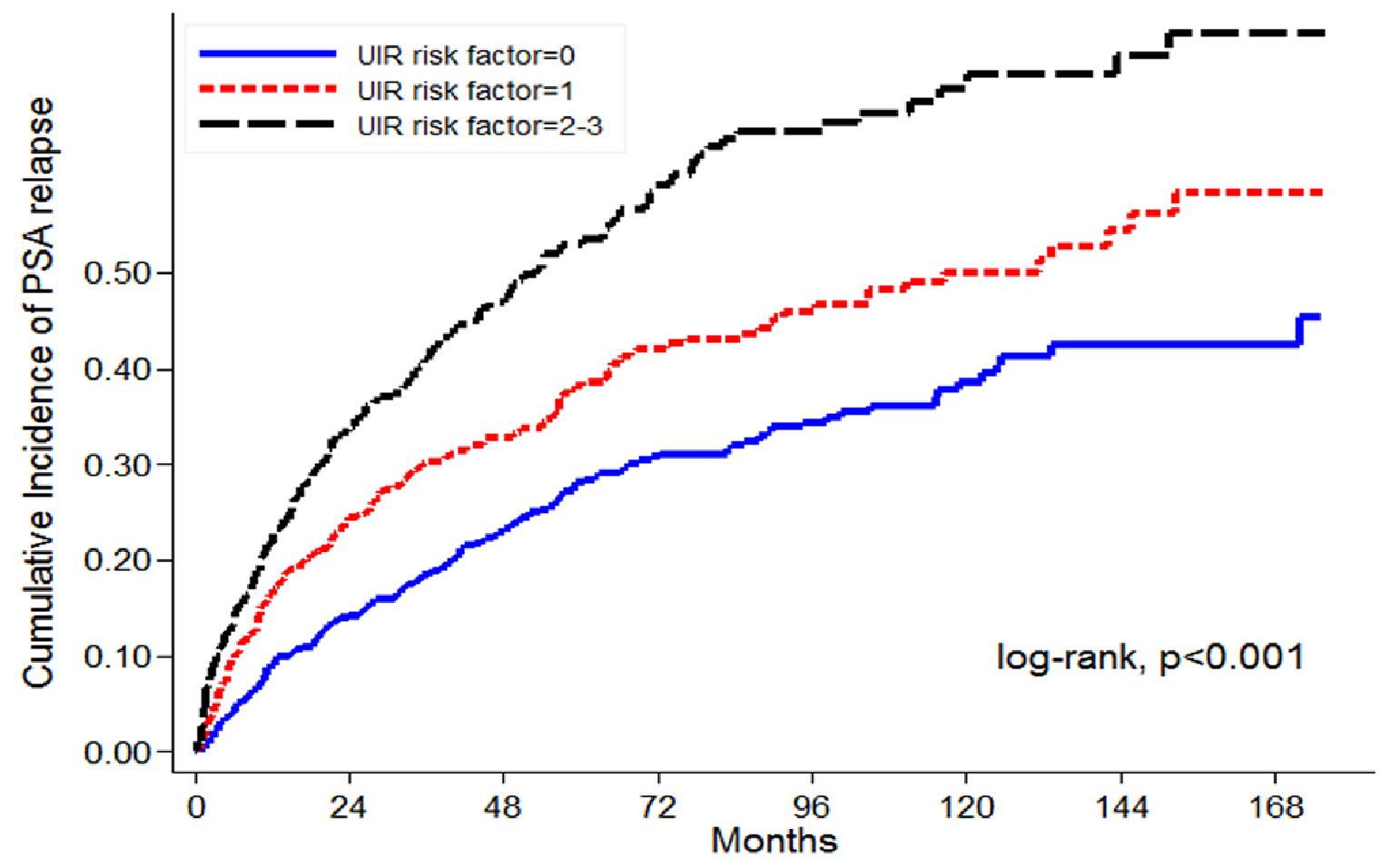

Fig 2A_300dpi . 


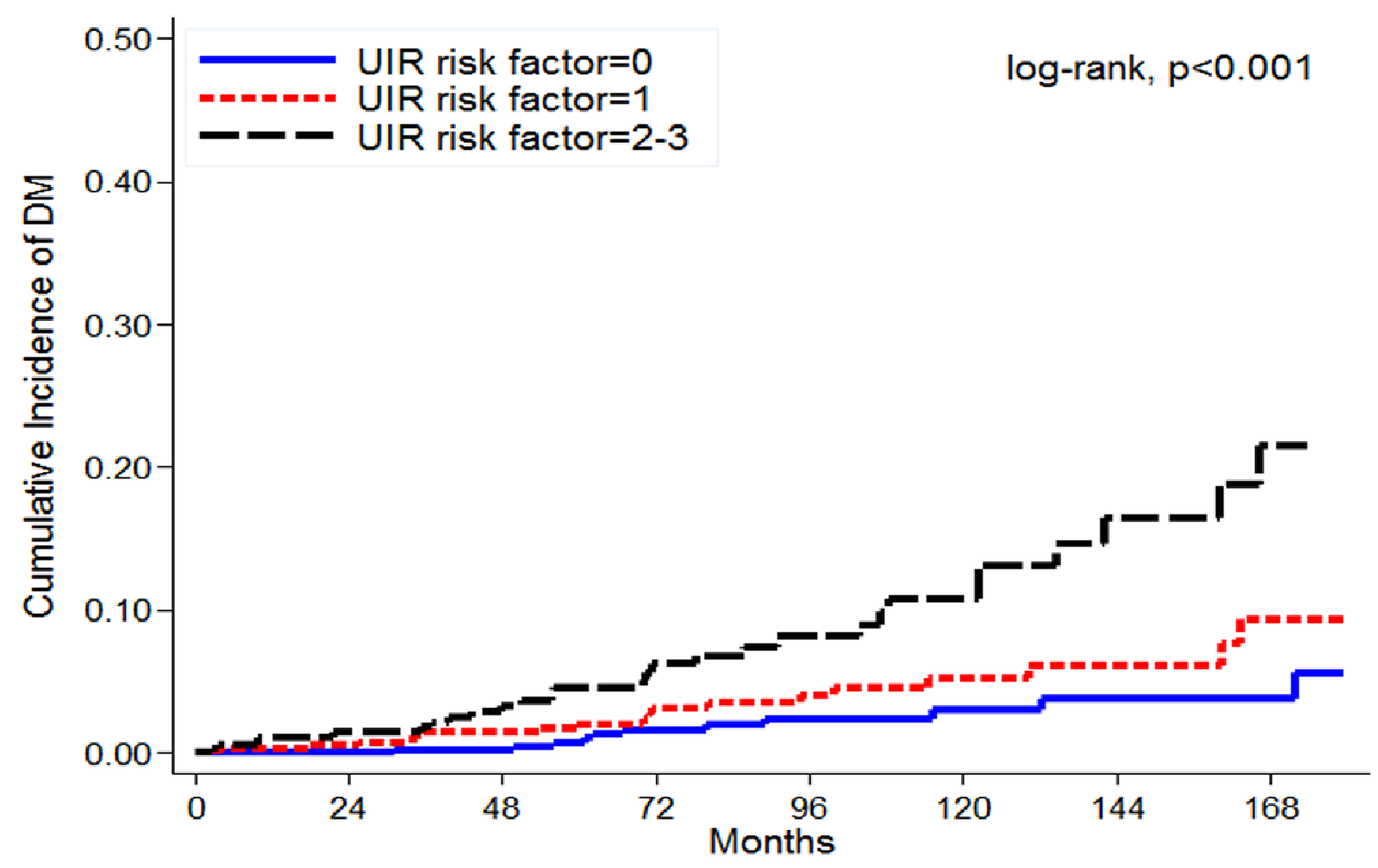

Fig 2B_300dpi . 


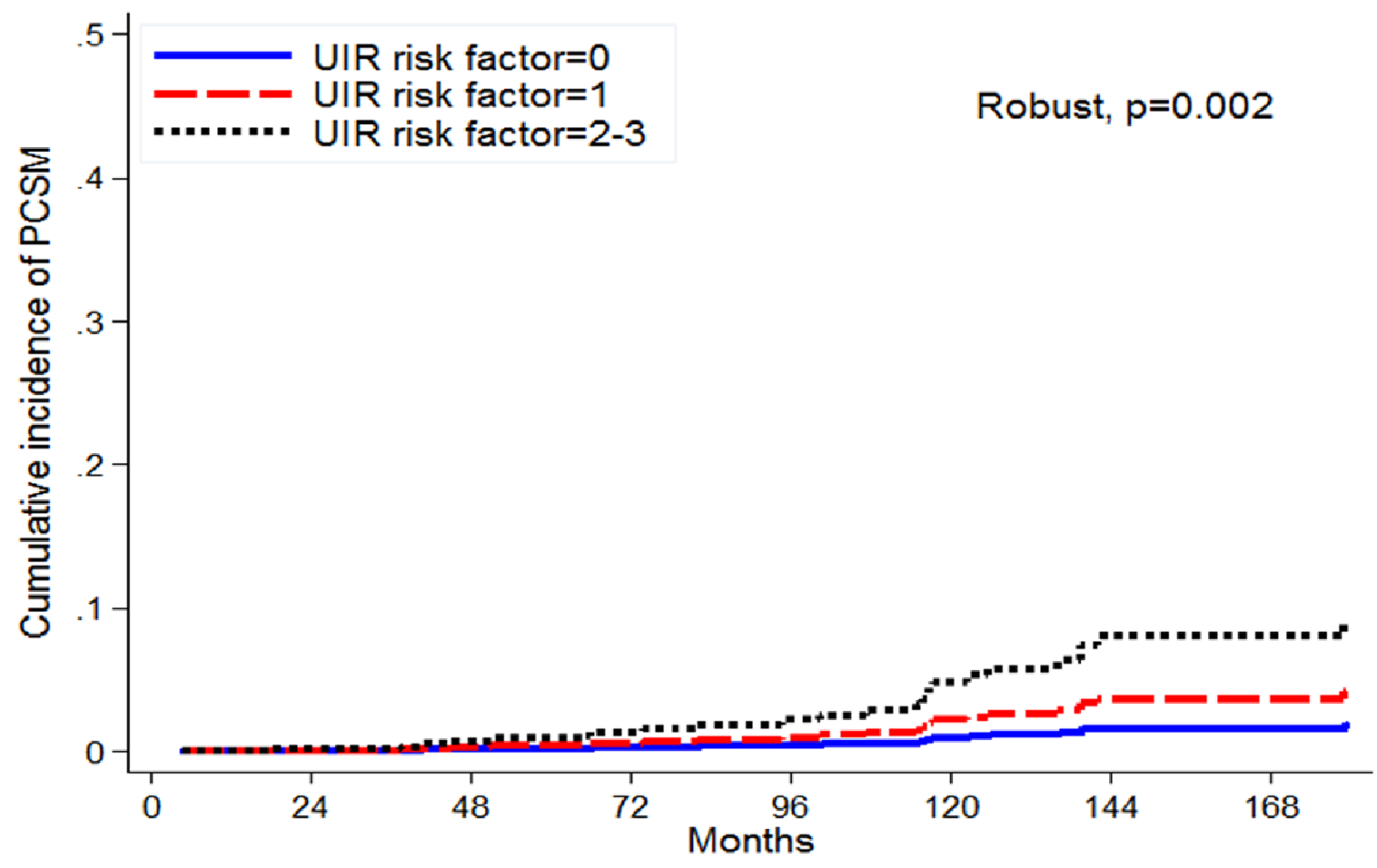

Fig 2C_300dpi . 


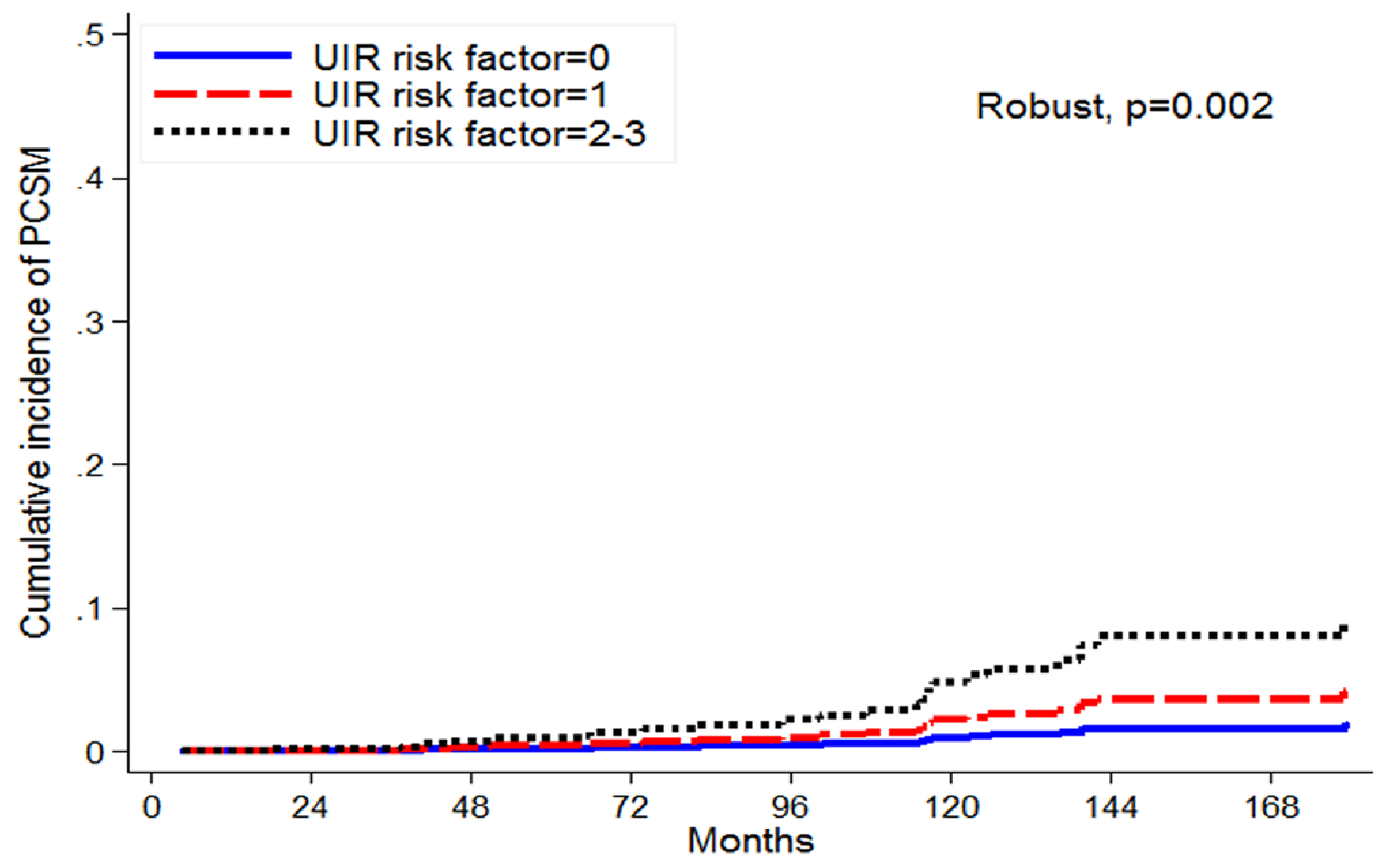

Fig 2C_300dpi . 


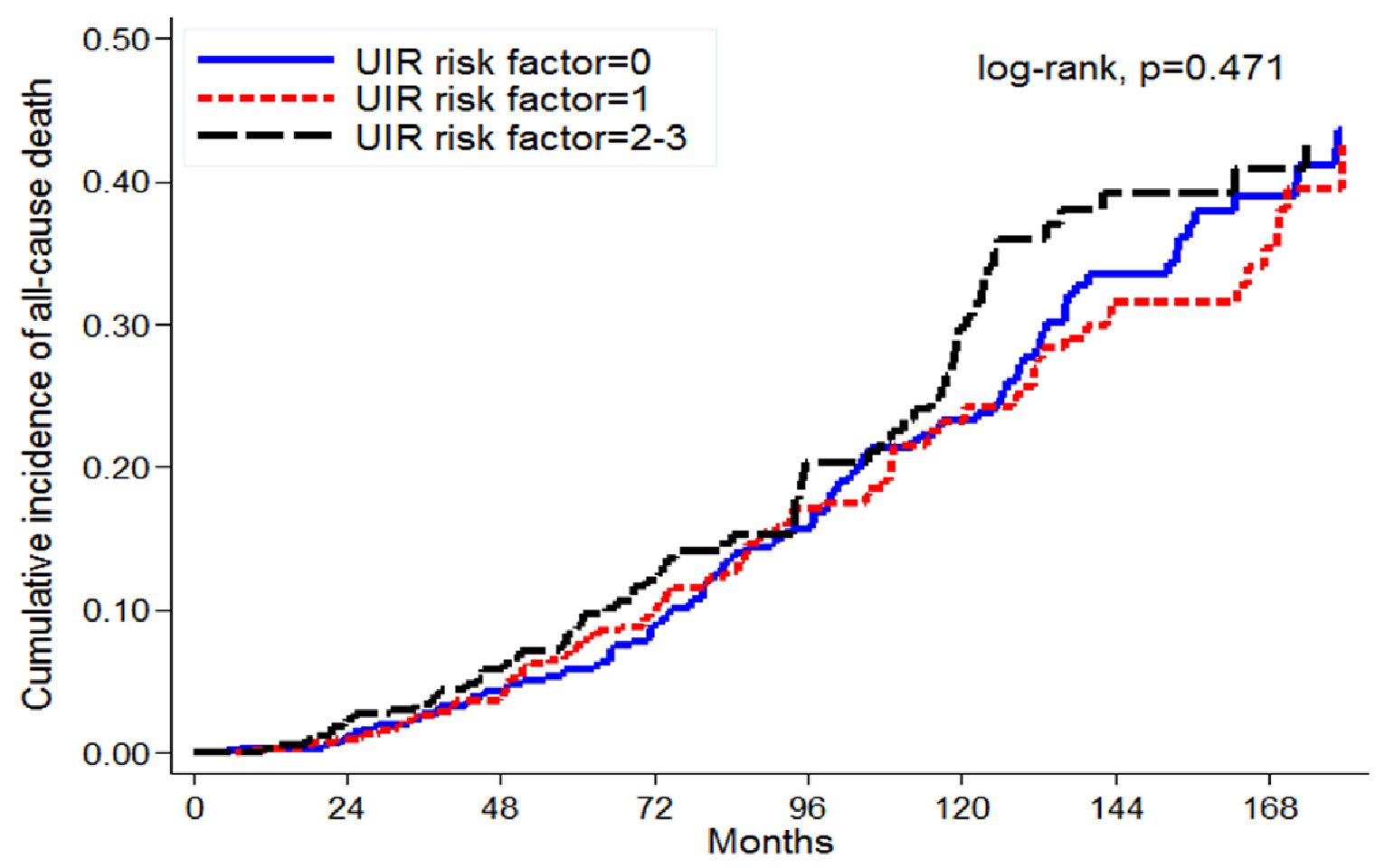

Fig 2D_300dpi . 